\title{
Design of Efficient CMOS Rectifiers for Integrated Piezo-MEMS Energy-Harvesting Power Management Systems
}

\author{
Martin Nielsen-Lönn, Prakash Harikumar, J Jacob Wikner, and Atila Alvandpour \\ Department of Electrical Engineering, Linköping University, SE-581 83 Linköping, Sweden \\ Emails: \{Martin.Nielsen.Lonn, Prakash.Harikumar, Jacob.Wikner, Atila.Alvandpour\}@liu.se
}

\begin{abstract}
MEMS-based piezoelectric energy harvesters are promising energy sources for future self-powered medical implant devices, low-power wireless sensors, and a wide range of other emerging ultra-low-power applications. However, the small form factors and the low vibration frequencies can lead to very low (in $\mu \mathrm{W}$ range) harvester output power. This makes the design of integrated CMOS rectifiers a challenge, ultimately limiting the overall power efficiency of the entire power management system. This work investigates two different fully integrated rectifier topologies, i.e. voltage doublers and full bridges. Implemented in $0.35-\mu \mathrm{m}, 0.18-\mu \mathrm{m}$, and $65-\mathrm{nm}$ CMOS technologies, the two rectifier architectures are designed using active diodes and crosscoupled pairs. These are then evaluated and compared in terms of their power efficiency and voltage efficiency for typical piezoelectric transducers in such ultra-low-power applications which generate voltages between 0.27-1.2 V. Furthermore, analytical expressions for the rectifiers are verified against circuit simulation results, allowing a better understanding of their limitations.
\end{abstract}

\section{INTRODUCTION}

Harvesting energy from the environment is an attractive choice for powering wireless sensors and medical implants. Utilizing piezoelectric (PE) transducers to extract energy from vibrations is particularly appealing due to its high energy density [1] and the wide availability of ambient vibration sources. A typical power management unit (PMU) in a PE harvester system is depicted in Fig. 1 which converts the alternating electrical output from the PE transducer into a steady voltage to supply a load circuit. The PMU has to maximize the power extracted from the PE transducer while supplying the specific voltage/current to the load circuit. Power consumption in the PMU must be minimized to limit energy loss. The focus of this work is on the rectifier sub-block of the PMU.

Consider the electrical equivalent circuit of a PE transducer shown in Fig. 2 [2]. Assuming a high $Q$ value, the opencircuit voltage $V_{P}=I_{P} / 2 \pi f_{P} C_{P}$ where $f_{P}$ is the frequency of vibration. In form-factor constrained applications with low ambient vibration levels (upto a few hundred $\mathrm{Hz}$ ), the output power and voltage $\left(V_{P}\right)$ generated by the PE transducer are typically low [3]. The design of CMOS rectifier circuits which provide high power and voltage efficiency at low operating voltages presents significant challenges. Several works which improve upon conventional rectifier topologies have been reported [2]-[5]. Unlike a majority of these works which utilize

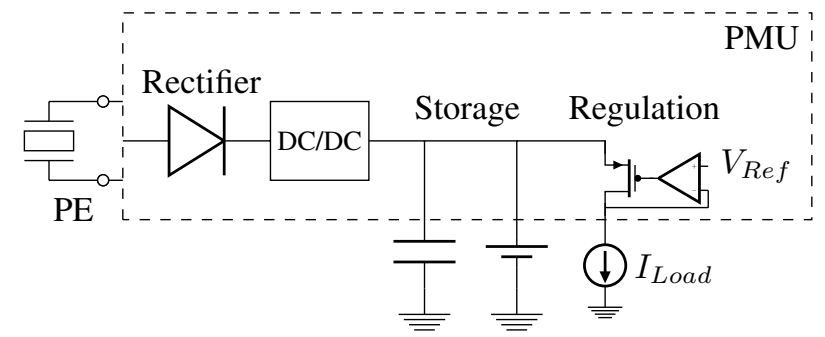

Fig. 1. Power management unit in a PE harvester system.

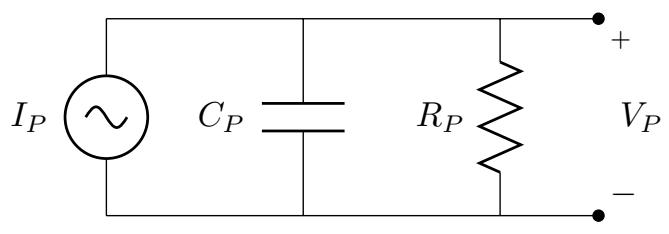

Fig. 2. Electrical equivalent circuit of a PE transducer.

$V_{P} \geq 1.2 \mathrm{~V}$, we have considered $V_{P}$ between $0.27-1.2 \mathrm{~V}$ that better represent $\mu \mathrm{W}$ PE transducers. Four variations of active rectifiers reported in literature have been simulated in $0.35-\mu \mathrm{m}$, $0.18-\mu \mathrm{m}$ and $65-\mathrm{nm}$ CMOS process technologies. Advantages and limitations of the selected rectifier topologies and CMOS technology nodes are illustrated based on the simulated power and voltage efficiency at specific values of $V_{P}$. The rest of the paper is organized as follows. Section II describes the rectifier topologies while Section III constitutes the analysis of the different topologies. Section IV compiles the simulation results and compares the performance of different rectifier topologies. Conclusions are drawn in Section V.

\section{RECTIFIER TOPOLOGIES}

Full-bridge rectifiers and voltage doublers are the two important types of rectifier circuits widely found in piezoelectric energy harvesting systems [2]. Different variants of these rectifiers have been simulated in this work. Since PN-junction diodes and diode-connected MOSFETs entail substantial voltage drops and consequently lower the power efficiency of the rectifier, they have been excluded. 


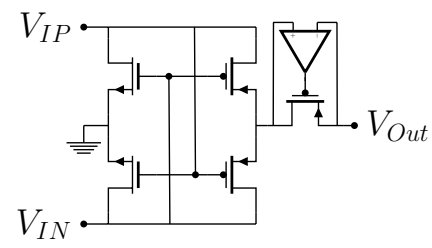

(a) NVC (XC)

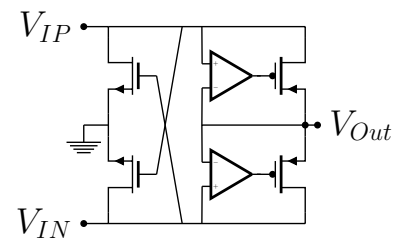

(c) Active PMOS XC NMOS (AP)

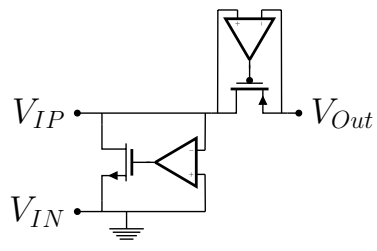

(b) Voltage doubler (VD)

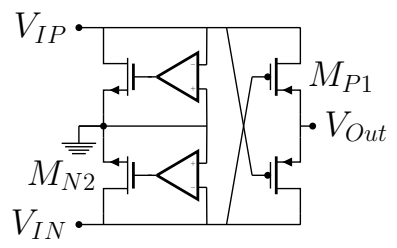

(d) Active NMOS XC PMOS (AN)
Fig. 3. Rectifier topologies.

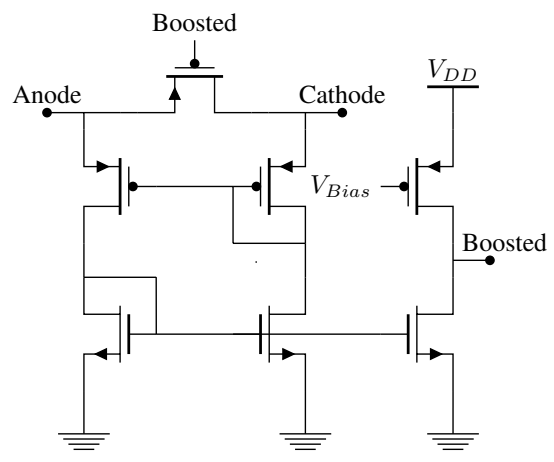

Fig. 4. Control circuitry with PMOS active diode.

\section{A. Full-bridge rectifiers}

Figure 3(a) illustrates a full-bridge rectifier which consists of a fully passive negative voltage converter (NVC) followed by an active diode [3]. When $V_{I P}$ goes high and $V_{I N}$ goes low, the top PMOS will have a positive $V_{S G}$ and conduct. The same occurs for the bottom NMOS which will have a positive $V_{G S}$ and conduct. This means that $V_{I N}$ will be connected to ground and $V_{I P}$ to $V_{O u t}$ through the active diode. Due to the cross-coupling of transistors, the NVC suffers only a very small voltage drop. In order to prevent the flow of reverse currents, the NVC is followed by an active diode.

The control circuitry used to implement the active diodes in the rectifiers should consume very low power to guarantee high power efficiency in the rectifiers. This work uses a biasfree comparator topology adopted from [6] which is shown in Fig. 4 along with the active PMOS diode. A simple commonsource amplifier has been added to generate a wide output swing. The comparator does not include any positive feedback or inverters after the output. Hence the comparator functions as an open-loop amplifier which significantly reduces the risk for oscillations. A complementary NMOS-input control circuit is utilized to control the active NMOS diodes.
For input voltages that are low in comparison to the threshold voltage of the PMOS devices, turning OFF the crosscoupled PMOS devices completely becomes difficult when the output voltage is higher than the input voltage. This results in a high leakage current which lowers the power efficiency. Figure 3(c) and Fig. 3(d) depict full-bridge rectifiers that utilize a combination of comparator-synchronized MOSFETs and cross-coupled MOSFETs. In Fig. 3(c), the active PMOS diodes mitigate reverse leakage currents by quickly turning OFF while the cross-coupled NMOS transistors entail a voltage drop of $V_{D S}$ only [7]. The rectifier shown in Fig. 3(d) employs crosscoupled PMOS diodes and active NMOS diodes.

\section{B. Voltage doublers}

A voltage doubler rectifier can achieve good power efficiency at higher output voltages compared to a full-bridge rectifier [2]. Figure 3(b) shows a voltage doubler employing active NMOS and PMOS diodes. When the input $V_{I P}$ drops below ground potential, the comparator controlling the NMOS device outputs a HIGH thus turning ON the NMOS device. When $V_{I P}$ becomes higher than the rectifier output voltage $V_{\text {Out }}$, the comparator controlling the PMOS device outputs a LOW thus turning ON the PMOS device and allowing $V_{\text {Out }}$ to charge towards $V_{I P}$.

\section{ANALYSIS}

Since the same analysis procedure applies to all the rectifier topologies, we have chosen the active NMOS cross-coupled PMOS rectifier shown in Fig. 3(d) as a representative example. Since the dominant voltage drop occurs across the turned-ON active NMOS diode, the voltage drop over conducting PMOS diodes are ignored. Consider a half-cycle of the PE transducer output during which $V_{I P}>V_{I N}$. During this half cycle the transistors $M_{P 1}$ and $M_{N 2}$ will be turned $\mathrm{ON}$. In this condition, $V_{I P}=V_{O u t}$ while $V_{I N}=V_{G N D}-V_{D S, M_{N 2}}-V_{O S}$ where $V_{O S}$ is the offset voltage of the comparator controlling $M_{N 2}$. The input voltage can be expressed as

$$
V_{P E}=V_{I P}-V_{I N}=V_{O u t}+V_{D S, M_{N 2}}+V_{O S} .
$$

Assuming that there is no leakage back to the PE transducer from $V_{O u t}$ and neglecting the parasitic capacitors in the circuit, the input voltage is obtained from charge conservation as

$$
V_{P E}=\frac{\frac{2 I_{P}}{\pi}-I_{\text {Load }}}{4 C_{P} f_{P}} .
$$

The time, $t_{0}$, required to charge $C_{P}$ to $V_{P E}$ is given by

$$
t_{0}=\frac{1}{\omega_{P}} \arccos \left(1-\frac{2 C_{P} V_{P E} \omega_{P}}{I_{P}}\right) .
$$

Since $M_{N 2}$ is turned $\mathrm{ON}$ by the wide-swing output of the comparator, its gate voltage is $\approx V_{D D}$ which places it in the triode region. Inserting (3) into the expression for drain current of a MOSFET in the triode region and solving for $V_{D S}$ gives

$$
V_{D S}=V_{E f f}-\sqrt{V_{E f f}^{2}-2 \frac{i_{P}\left(t_{0}\right)}{\mu_{n} C_{o x} \frac{W}{L}}}
$$




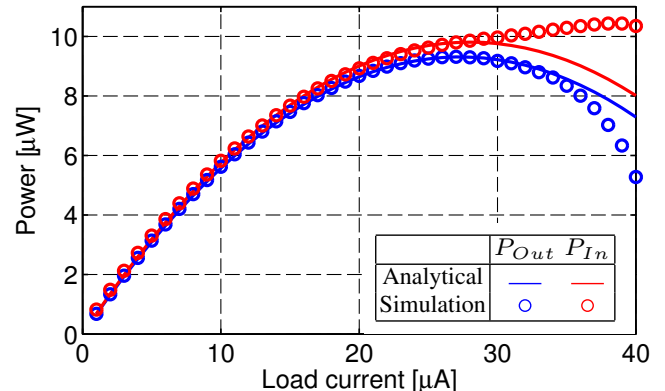

Fig. 5. $P_{I n}$ and $P_{\text {Out }}$ for the Active NMOS XC PMOS rectifier in $0.18-\mu \mathrm{m}$.

where $V_{E f f}=V_{D D}-V_{T h, n}$ for the active NMOS diode. The power efficiency which is defined as the ratio of output power, $P_{\text {Out }}$, to input power, $P_{I n}$, can be calculated as

$$
\eta_{P}=\frac{P_{\text {Out }}}{P_{\text {In }}}=\frac{I_{\text {Load }} V_{\text {Out }}}{V_{P E}\left(\frac{2 I_{P}}{\pi}-4 C_{P} V_{P E} f_{P}\right)} .
$$

Figure 5 shows the plot of $P_{O u t}$ and $P_{I n}$ from (5) along with the corresponding values from simulation for the $0.18-\mu \mathrm{m}$ AN rectifier with $I_{P}=88 \mu \mathrm{A}$. A comparator with an offset of $17 \mathrm{mV}$ was used. The discrepancy for higher load currents is caused by the low $V_{P E}$ which increases the voltage drop across the cross-coupled PMOS diodes.

\section{Simulation Results}

In this paper we consider the three scenarios shown in Table I which correspond to different levels of input acceleration on the PE transducer. At higher acceleration, higher values of $I_{P}$ and $V_{P}$ are obtained. The rectifier topologies in Fig. 3 were simulated for the $I_{P}$ values listed in Table I with a constant current load and a $1 \mu \mathrm{F}$ smoothing capacitor connected to the output node $V_{\text {Out }}$. For each value of $I_{P}$, the load current of the rectifier was swept in order to determine the maximum load current provided by the rectifier. The variation in power and voltage efficiency of the rectifier with the load current was determined. The selected CMOS process nodes differ significantly in their nominal supply voltage $V_{D D}$, threshold voltage $V_{T h}$, and the intrinsic gain of the MOS transistors. $V_{T h}$ plays a major role in determining the minimum input voltage $\left(V_{P}\right)$ supported by a rectifier while the intrinsic gain and transconductance are crucial in determining the performance at large load currents. Since the control circuitry for the active diodes use the nominal supply voltage, their performance is significantly impacted by the choice of the CMOS process.

All figures in this section use the acronyms for the rectifiers provided in Fig. 3 to distinguish each rectifier topology. Figures $6-8$ plot the variation in power efficiency of the rectifiers

TABLE I

PE TRANSDUCER MODEL PARAMETERS FOR DIFFERENT ACCELERATIONS.

\begin{tabular}{c|c|c|c|c}
$I_{P}[\mu \mathrm{A}]$ & $C_{P}[\mathrm{nF}]$ & $R_{P}[\mathrm{M} \Omega]$ & $f_{P}[\mathrm{~Hz}]$ & $V_{P}[\mathrm{~V}]$ \\
\hline 34 & 100 & 100 & 200 & 0.271 \\
88 & 100 & 100 & 200 & 0.7 \\
150 & 100 & 100 & 200 & 1.19
\end{tabular}

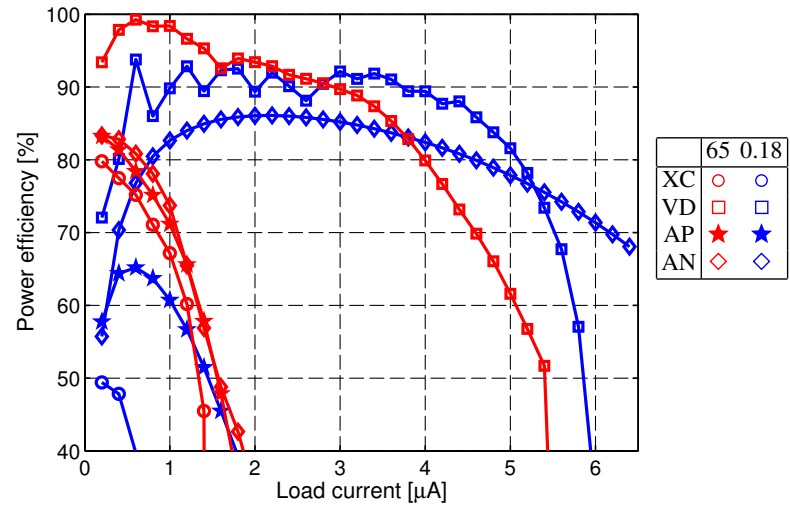

Fig. 6. Power efficiency for $I_{P}=34 \mu \mathrm{A}$.

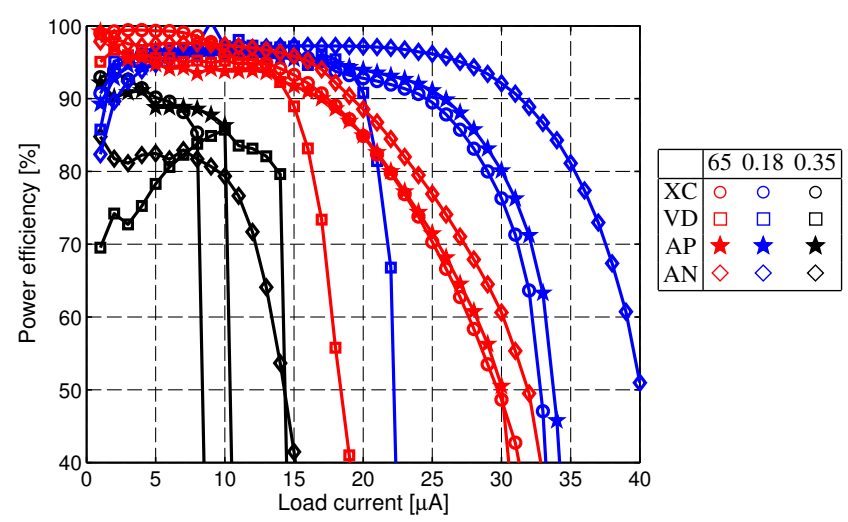

Fig. 7. Power efficiency for $I_{P}=88 \mu \mathrm{A}$.

with load current for the specified values of $I_{P}$. The corresponding plots for voltage efficiency are shown in Figs. 911. For the voltage doublers, the voltage efficiency has been computed as $V_{O u t} / 0.5 V_{P E}$. For $I_{P}=34 \mu \mathrm{A}$, the rectifiers simulated in more advanced CMOS process nodes achieve higher power and voltage efficiency while those simulated in $0.35-\mu \mathrm{m}$ never get activated since $V_{P}=271 \mathrm{mV}$ is insufficient to turn $\mathrm{ON}$ the transistors which have a $V_{T h} \approx 0.6 \mathrm{~V}$. When the load current increases, resulting in a lower $V_{O u t}$, the higher intrinsic gain of the $0.18-\mu \mathrm{m}$ transistors provides increased efficiency over a wider load interval as illustrated in the power and voltage efficiency plots. Even though the $0.35-\mu \mathrm{m}$ simulations are functional at $I_{P}=88 \mu \mathrm{A}$, the poor transconductance of the devices stemming from low gateoverdrive voltage leads to diminished efficiency at medium and high load currents as shown in Figs. 7 and 10. For $I_{P}=$ $150 \mu \mathrm{A}$, the $65-\mathrm{nm}$ and $0.18-\mu \mathrm{m}$ rectifiers maintain substantial power and voltage efficiency even at fairly large load currents. From the plots in Figs. 6-11, it is seen that the AN rectifier sustains higher efficiency over a wider range of load currents compared to other topologies since the comparator for the active NMOS diode utilizes the full nominal supply voltage of the CMOS process. In contrast, the comparator for the active PMOS diode shown in Fig. 4 uses the rectifier input and output voltage as its supply voltage. In the power and voltage 
efficiency plots, some of the curves experience a steep decline which can attributed to the control circuitry becoming nonfunctional due to low $V_{O u t}$ at higher load currents. To provide

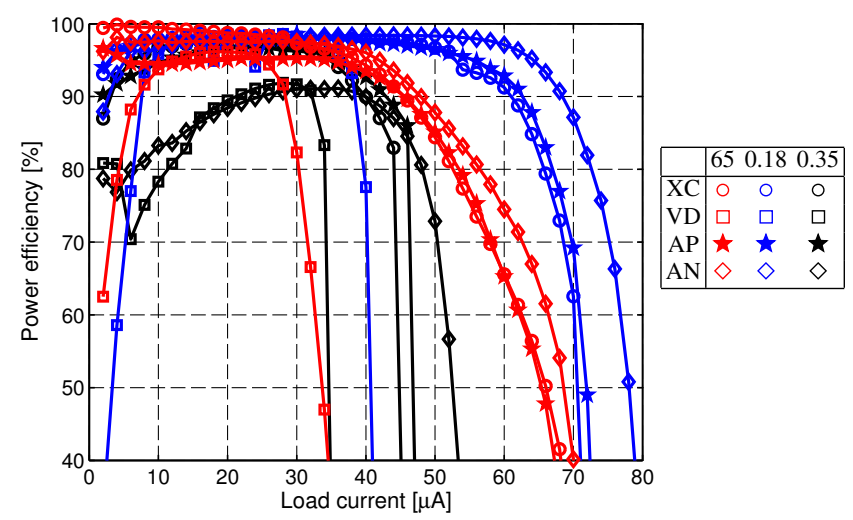

Fig. 8. Power efficiency for $I_{P}=150 \mu \mathrm{A}$.

a fair comparison between the different CMOS processes and $I_{P}$ values employed, the quiescent power consumption of the control circuits was not included in the calculation of the power efficiency.

\section{CONCLUSiON}

Rectifier topologies which are suitable for extracting energy from $\mu \mathrm{W}$-range $\mathrm{PE}$ transducers that generate open-circuit voltages in the range of $0.27-1.2 \mathrm{~V}$ have been investigated. Analytical expressions for the rectifier input power and output power have been verified against simulated values and found to match closely. The selected rectifier topologies have been simulated in three CMOS process nodes which have substantially different process parameters. Based on the variation in power and voltage efficiency across load currents, the merits and drawbacks of the selected rectifier topologies and CMOS processes are elucidated.

\section{ACKNOWLEDGMENT}

This project has received funding from the European Union's Horizon 2020 research and innovation programme

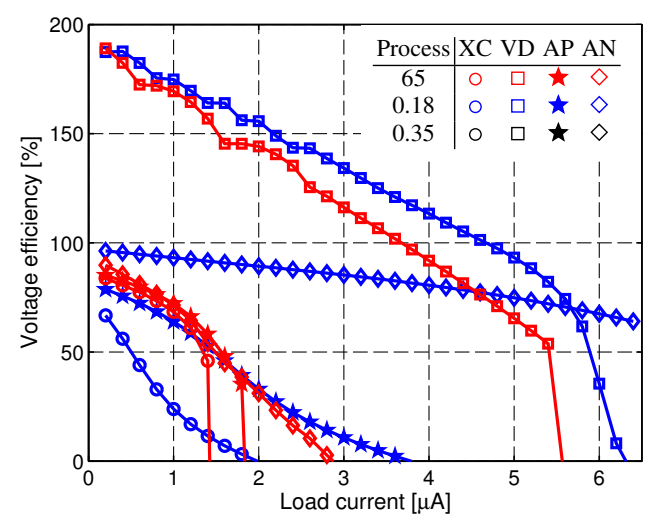

Fig. 9. Voltage efficiency for $I_{P}=34 \mu \mathrm{A}$.

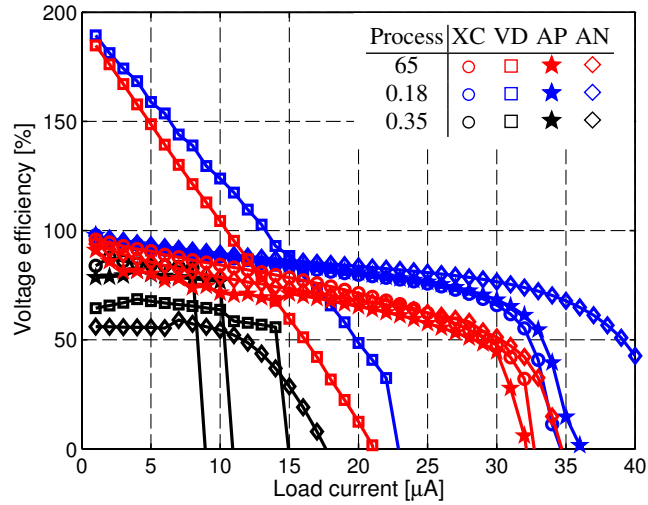

Fig. 10. Voltage efficiency for $I_{P}=88 \mu \mathrm{A}$.

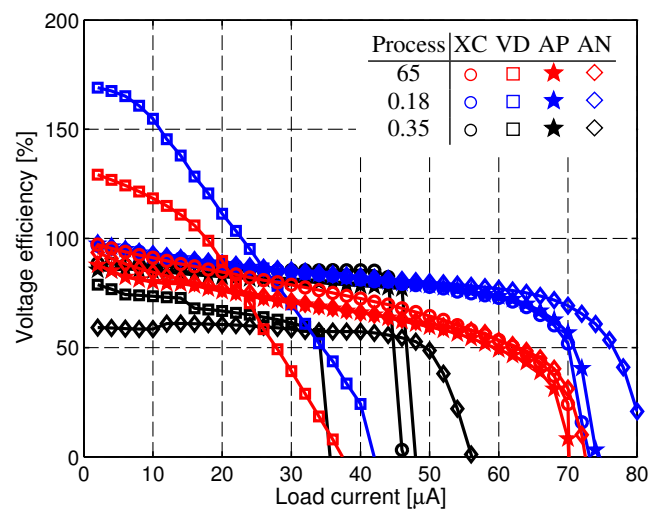

Fig. 11. Voltage efficiency for $I_{P}=150 \mu \mathrm{A}$.

under grant agreement No 644378. It is also supported by Sweden's Innovation Agency, VINNOVA. Furthermore, we would like to thank our project partners, specially Dr. Cristina Rusu (Acreo Swedish ICT AB) and Dr. Thorbjörn Ebefors (Silex Microsystems AB) for valuable discussions.

\section{REFERENCES}

[1] S. Roundy and P. K. Wright, "A piezoelectric vibration based generator for wireless electronics," Smart Materials and Structures, vol. 13, no. 5, pp. 1131-1142, Aug. 2004.

[2] Y. Ramadass and A. Chandrakasan, "An efficient piezoelectric energy harvesting interface circuit using a bias-flip rectifier and shared inductor," IEEE J. Solid-State Circuits, vol. 45, no. 1, pp. 189-204, Jan. 2010.

[3] C. Peters, J. Handwerker, D. Maurath, and Y. Manoli, "A sub-500 mV highly efficient active rectifier for energy harvesting applications," IEEE Trans. Circuits Syst. I, vol. 58, no. 7, pp. 1542-1550, July 2011.

[4] S. Guo and H. Lee, "An efficiency-enhanced CMOS rectifier with unbalanced-biased comparators for transcutaneous-powered high-current implants," IEEE J. Solid-State Circuits, vol. 44, no. 6, pp. 1796-1804, June 2009.

[5] Y. Sun, N. H. Hieu, C.-J. Jeong, and S.-G. Lee, "An integrated highperformance active rectifier for piezoelectric vibration energy harvesting systems," IEEE Trans. Power Electron., vol. 27, no. 2, pp. 623-627, Feb. 2012.

[6] C. van Liempd, S. Stanzione, Y. Allasasmeh, and C. Van Hoof, "A $1 \mu \mathrm{W}$-to-1 mW energy-aware interface IC for piezoelectric harvesting with $40 \mathrm{nA}$ quiescent current and zero-bias active rectifiers," in ISSCC Dig. Tech. Papers, Feb. 2013, pp. 76-77.

[7] S. T. Kim, T. Song, J. Choi, F. Bien, K. Lim, and J. Laskar, "Semi-active high-efficient CMOS rectifier for wireless power transmission," in IEEE RFIC Symp., May 2010, pp. 97-100. 